J. Clin. Chem. Clin. Biochem.

Vol. 19, 1981, pp. $997-1000$

\title{
Teststreifen für Screening-Untersuchungen zum semiquantitativen Nachweis von Proteinurien
}

\author{
Von D. Vonderschmitt und $A$. Scholer \\ Zentrallabor des Kantonsspitals Basel (Schweiz)
}

(Eingegangen am 6. September 1980/12. Januar 1981)

Zusammenfassung: Marktübliche Streifentests, die den Eiweißfehler bestimmter Indikatoren für den kolorimetrischen Nachweis von Protein (speziell Albumin) nutzen, werden mit einer quantitativen nephelometrischen Methode verglichen. Die Untersuchung zeigte eine gute Übereinstimmung zwischen quantitativer Methode (Automated Immunoprecipitin) und Aussage der Teststreifen (Rapignost Protein/Combur-8-Test). Insgesamt trennten beide Teststreifen die Segmente für die semiquantitativen Bestimmungen gut. Zwischen den Teststreifen ergab sich in proteinhaltigen Urinen eine sehr gute Úbereinstimmung. In proteinfreien Urinen zeigte der Combur-8-Test einen geringfügig höheren Anteil falsch-positiver Resultate.

\section{Evaluation of test strips for the semi-quantitative screening for proteinurias}

Summary: Commercial test strips using the protein error of certain indicators for the colorimetric detection of protein (especially albumin) were compared with a quantitative nephelometric method.

The study proved that there is good agreement between the results from the quantitative method and those from the test strips (Rapignost Protein/Combur-8-Test). Both test strips generally showed a good separation of the segments for semi-quantitative determinations. There was a very good agreement between the test strips in protein-containing urines. In protein-free urines, however, the Combur-8-Test showed a slightly higher proportion of false-positive results.

\section{Einfüihrung}

Chronische Nierenkrankheiten erfordern in den Endstadien erhebliche Aufwendungen für operative und Heilbehandlungen. Die Morbiditätsrate wird aus epidemiologischen Studien mit 3,5\% für chronische Nephropathien angegeben (1). Eine deutliche Verbesserung der Prognose und Reduktion der Aufwendung ist durch frühzeitige Erkennung und intensive nephrologische Betreuung zu erzielen.

Als leicht nachweisbares häufiges Symptom bei Nierenerkrankungen kommt die Proteinurie in Betracht, wobei zu berụ̈cksichtigen ist, daß auch körperliche und nervliche Belastung.(Streß) sowie Fieber eine Erhöhung der physiologischen Proteinausscheidung bewirken können (2-6). Eine Verbesserung der diagnostischen Spezifität wird durch mehrmalige Wiederholung des Nachweises einer Proteinurie erreicht (1).

Hierfür eignen sich insbesondere die einfach zu handhabenden, billigen Streifentests $(7,8)$. Im folgenden werden daher anhand 440 Patientenurinen zwei marktübliche Proteinteststreifen mit einer quantitativen Proteinbestimmung verglichen.

\section{Versuchsanordnung und -durchfithrung}

Es wurden 440 Urinproben untersucht, welche an 10 aufeinanderfolgenden Tagen im Routinelabor des klinisch-chemischen Laboratoriums des Kantonsspitals Basel eintrafen. Eine spezielle Auswahl der Urine fand nicht statt. Fünf Personen haben am Test teilgenommen: Eine Laborantin, zwei Arztgehilfinnen, zwei Studenten. Zum Vergleich wurden die Teststreifen Combur-8-Test der Firma Boehringer Mannheim (abgekürzt mit Combur) und Rapignost Protein der Firma Behringwerke AG (abgekürzt mit Rapignost) herangezogen. Dic Ablesung der Teststreifen erfolgte teils bei Tageslicht, teils bei Kunstlicht. Als Referenzmethode diente die „Automated Immunoprecipitin Methode" (Gesamt-Serumeiweiß) der Firma Technicon, im folgenden abgekürzt mit AIP.

Das Nachweisprinzip der beiden Streifentests beruht auf dem bercits 1909 von Soerensen beschriebenen und 1937 von Feigl \& Anger (9) erstmals verwende ten Eiweißfehler bestimmter Indikatorfarbstoffe. Der Rapignoststreifen enthält auf cinem sauer gepufferten Testfeld den Indikator Tetrabromphenolblau, der Comburstreifen Tetrachlor-tetrabromsulphophthalein. Beide Indikatoren zeigen mit steigendem Protein- (speziell Albumin(10))gehalt eine Farbverschiebung von gelb nach grün-blau.

Dic Referenzbestimmungen wurden auf einem Auto-Analyzer ${ }^{\circledR}$ mit Nephclometer nach Angaben von Killingsworth et al. Jurchgeführt (11).

Um die Gesamtheit der im Urin ausgeschiedenen Surum-Proteine zu erfassen, wurde ein polyspezifisches Antihuman-Serum (DAKOPATT, Kopenhagen) zur Immun-Präzipitation verwen- 
det. Diese Methode wird im klinisch-chemischen Laboratorium des Kantonsspitals Basel seit drei Jahren verwendet. Sie spricht nicht auf Bence-Jones-Proteine und Mukoproteine an. Ihre Resultate stimmen mit den klinischen Befunden besser überein als die früher übliche Kjeldahl-Bestimmung der Proteine (12).

\section{Ergebnisse}

Von den untersuchten 440 Urinen lagen etwa 2/3 im Normalbereich der Proteinkonzentration (0-150 mg/l Protein im Urin), etwa $1 / 5$ im Bereich $150-1000 \mathrm{mg} / 1$ und knapp $7 \%$ darüber. Die Verteilung der quantitativ ermittelten Proteinkonzentrationen zeigte ein deutliches Maximum im Bereich 0-150 mg/l.

Tab. 1. Relative Verteilung der untersuchten Urine auf Proteinkonzentrationsbereiche. Die Abgrenzung der Konzentrationsbereiche erfolgte nach Abstufungen der Teststreifenvergleichsskalen.

\begin{tabular}{lcr}
\hline $\begin{array}{l}\text { Proteinkonzentrationsbereich } \\
\text { gemäß AIP } \\
\text { (mg/l) }\end{array}$ & $\begin{array}{l}\text { Anzahl } \\
\text { der Urine }\end{array}$ & Anteil \\
\hline 0 & & $(\%)$ \\
$>\quad 0-150$ & 57 & 13,0 \\
$>150-300$ & 260 & 59,1 \\
$>300-1000$ & 47 & 10,7 \\
$>1000-5000$ & 46 & 10,5 \\
$>5000$ & 22 & 5,0 \\
& 8 & 1,8 \\
& 440 & 100,1 \\
\hline
\end{tabular}

Nach Zuordnung der erhaltenen Teststreifenbefunde zu den quantitativ ermittelten Konzentrationen wurde die Häufigkeitsverteilung der Teststreifenanzeigen in den nach Tabelle 1 vorgegebenen Konzentrationsbereichen errechnet. Die resultierenden Werte zeigen Tabelle 2 und Abbildung 1. Eine sehr gute Übereinstimmung zwischen quantitativer und Teststreifenaussage ergab der Rapignoststreifen in den Bereichen $0 \mathrm{mg} / 1$ und $150-300 \mathrm{mg} / 1$ mit nur etwa $9 \%$ falsch-positiven und etwa $6 \%$ falschnegativen Anzeigen. Weniger gut zeigte sich hier der Comburstreifen mit etwa 19\% falsch-positiven und etwa 9\% falsch-negativen Anzeigen. In den Bereichen 3001000 und $1000-5000 \mathrm{mg} / \mathrm{l}$ fand erwartungsgemäß eine Verteilung der Anzeigen auf die Meßpunkte 300/1000 bzw. 1000/5000 statt. Die Übereinstimmung zwischen quantitativer und Teststreifenaussage sowie der Teststreifen untereinander war ebenfalls gut. Konzentrationen über $5000 \mathrm{mg} / 1$ Urin wurden ausnahmslos richtig angezeigt.

Im folgenden wurde die Häufigkeitsverteilung der Teststreifenanzeigen in den Meßpunkten der Teststreifenskala zugeordneten Konzentrationsbereichen errechnet. Die Zuordnung erfolgte in der durch eine Versuchsreihe bestätigten Annahme einer nicht linearen Abhängigkeit des subjektiven Farbeindrucks von der durch Teststreifen angezeigten Proteinkonzentration. Dem Meßpunkt $300 \mathrm{mg} / 1$ entspricht demnach der Bereich $150-500 \mathrm{mg} / \mathrm{l}$, Meßpunkt $1000 \mathrm{mg} / 1$ entspricht $500-2500 \mathrm{mg} / 1$ und Meßpunkt 5000 mg/l entspricht 2500-5000 mg/l, wobei die Grenzen 500 bzw. 2500 mg/l die Konzentration sind, bei denen der subjektiv empfundene Farbton gerade genau zwischen dem tieferen und höheren Meßpunkt der Vergleichsșkala liegt.

Die übrigen Bereiche wurden aus Tabelle 1 übernommen. Das Ergebnis dieser Häufigkeitsverteilung zeigt Abbildung 2, deren Aufbau dem der Abbildung 1 entspricht.

\section{Diskussion}

Das Prinzip des Proteinnachweises mittels Indikatoren, deren Eiweißfehler einen sichtbaren Farbumschlag bei Anwesenheit von Eiweiß hervorrufen, wird heute bei allen bekannten Streifentests angewandt $(7,13)$. Aus der hohen Affinität solcher Indikatoren gegenüber freien Aminogruppen resultiert, unabhängig vom Indikator, eine sehr hohe Empfindlichkeit gegenüber Albumin, dessen Ausscheidung ein wesentliches Indiz für Nierenerkrankungen darstellt.

Man muß jedoch berücksichtigen, daß diese Indikatoren Globuline sehr viel weniger empfindlich und Bence-

Jones-Proteine nicht anzeigen $(10,12)$. Die Referenzmethode erfaßt hingegen die Globuline. Diese Tatsache kann zur Erklärung einzelner falsch-negativer Streifentests herangezogen werden. Andererseits ist bekannt, daß größere Konzentrationen von Mukoproteinen die Teststreifen verfärben, durch die Referenzmethode jedoch nicht erfaßt werden und deshalb im Vergleich der beiden Methoden zu falsch-positiven Testergebnissen

Tab. 2. Häufigkeitsverteilung von Teststreifen angezeigter Konzentrationsstufen in jeweiligen Konzentrationsbereichen (gemäß AIP). (Konzentrationsstufe gemäß Farbskala (mg/l).

\begin{tabular}{|c|c|c|c|c|c|c|c|c|c|c|}
\hline \multirow{2}{*}{$\begin{array}{l}\text { Proteinkonzentration } \\
\text { gemäß AIP } \\
(\mathrm{mg} / \mathrm{l})\end{array}$} & \multicolumn{5}{|c|}{$\begin{array}{l}\text { Rapignost } \\
\text { (Angaben in \%) }\end{array}$} & \multicolumn{5}{|c|}{$\begin{array}{l}\text { Combur } \\
\text { (Angaben in \%) }\end{array}$} \\
\hline & $0-150$ & 300 & 1000 & 5000 & $\Sigma$ & neg. & 300 & 1000 & 5000 & $\Sigma$ \\
\hline $\begin{array}{l}0 \\
0-150 \\
150-300 \\
300-1000 \\
1000-5000 \\
5000\end{array}$ & $\begin{array}{l}91,2 \\
54,6 \\
6,4 \\
- \\
- \\
-\end{array}$ & $\begin{array}{r}7,0 \\
45,4 \\
80,9 \\
52,2 \\
9,1 \\
-\end{array}$ & $\begin{array}{l}1,8 \\
- \\
10,6 \\
45,7 \\
40,9 \\
-\end{array}$ & $\begin{array}{r}- \\
- \\
2,1 \\
2,2 \\
50,2 \\
100,0\end{array}$ & $\begin{array}{l}100,0 \\
100,0 \\
100,0 \\
100,1 \\
100,0 \\
100,0\end{array}$ & $\begin{array}{l}80,7 \\
53,8 \\
8,5 \\
- \\
- \\
-\end{array}$ & $\begin{array}{c}19,3 \\
45,3 \\
78,7 \\
60,0 \\
4,5 \\
-\end{array}$ & $\begin{array}{r}- \\
0,8 \\
.10,6 \\
37,0 \\
40,9 \\
-\end{array}$ & $\begin{array}{r}- \\
-\quad 2,1 \\
2,2 \\
54,5 \\
100,0\end{array}$ & $\begin{array}{r}100,0 \\
99,9 \\
99,9 \\
100,1 \\
99,9 \\
100,0\end{array}$ \\
\hline
\end{tabular}




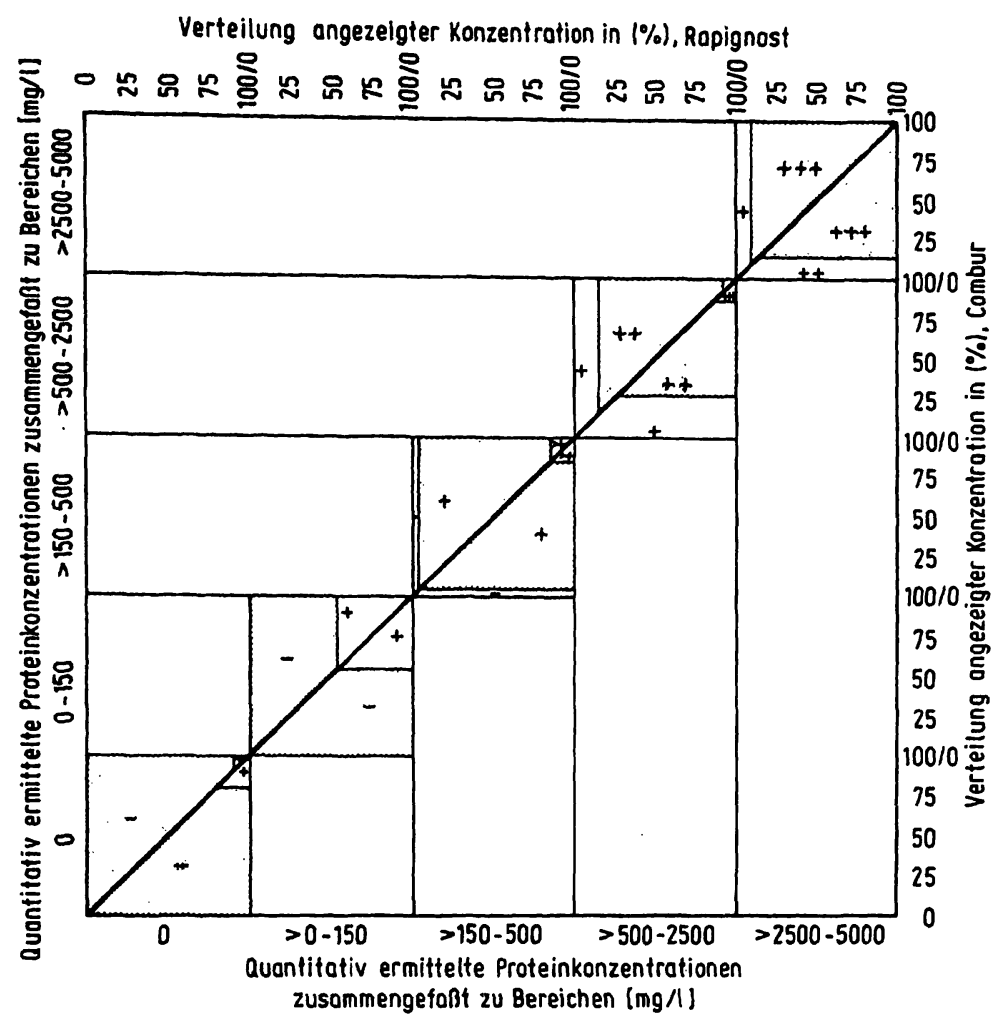

Abb. 1. Relative Verteilung der Teststreifenanzeige auf quantitativ ermittelte Konzentrationen.

In diesem Diagramm sind dic Werte der Tabelle 2 zusammengefaßt. Die Kästchen obcrhalb der $45^{\circ}$-Geraden sind Rapignost, die darunterliegenden Combur zuzuordnen. Die Symbole $-1+1++/+++$ entsprechen den Farbskalafeldern 0-150 bzw. neg./ $300 / 1000 / 5000$.

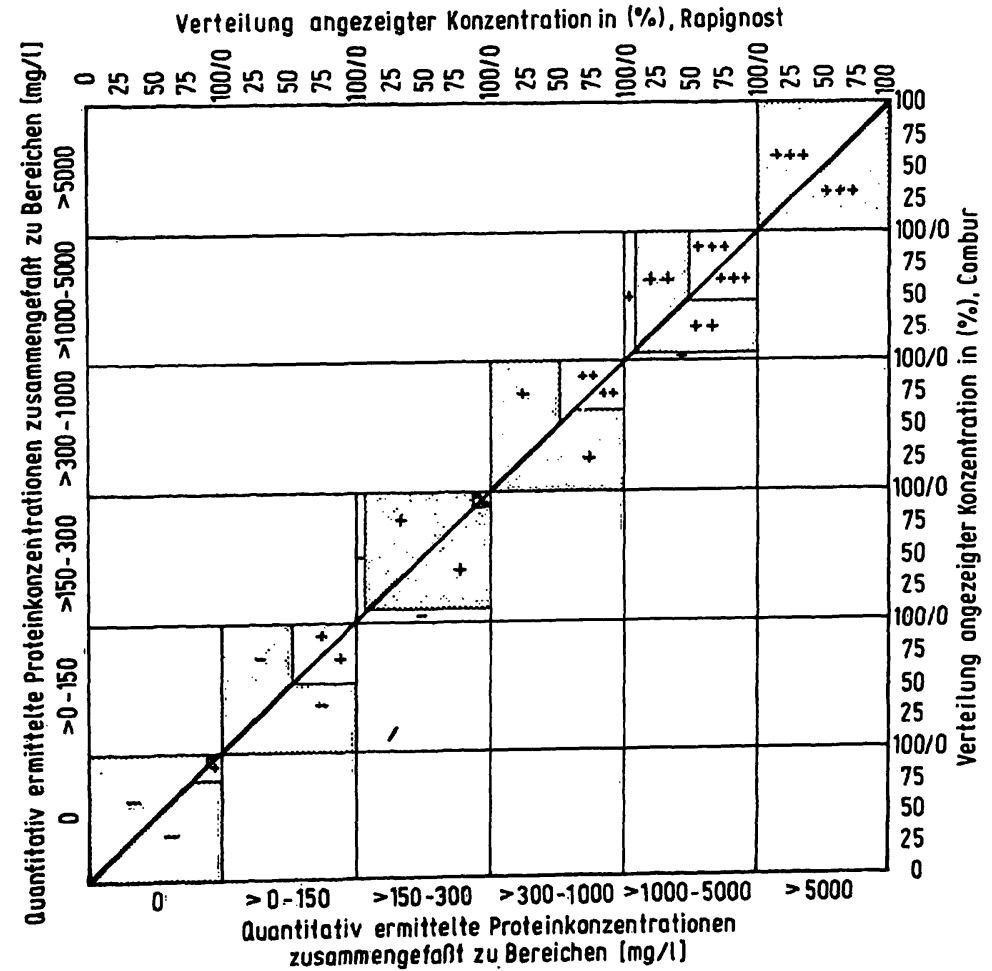

Abb. 2. Häufigkeitsverteilung der Teststreifenanzeigen aus Abb. 1. 
führen. Die vorgelegten Resultate bestätigen allerdings, daß die Unterschiede nicht ins Gewicht fallen. Die Untersuchung zeigte speziell in dem furr Notfalldiagnostik und Screeninguntersuchungen interessanten Konzentrationsbereich oberhalb $150 \mathrm{mg} / \mathrm{l}$ Protein im Urin eine zufriedenstellende Übereinstimmung zwischen quantitativer und Teststreifenaussage.

In den Bereichen $>300-1000$ und $>1000-5000 \mathrm{mg} / 1$ wurde erwartungsgemäß eine Auftrennung der Teststreifenanzeigen auf die Konzentrationsstufen 300/ 1000 bzw. 1000/5000 der Farbvergleichsskalen erhalten.

Unter Berücksichtigung eines nichtlinearen Zusammenhangs zwischen visuellem Farbeindruck und Protein-

\section{Literatur}

1. Tredt, H.-J., Stolze, G. \& Friedemann, H. (1974) Dtsch. Gesundheitswes. 29, 488-494.

2. Hepler, O. E. (1970) Manual of Clinical Laboratory Methods, 4 th Ed., S. 6, Verlag C. C. Thomas, Springfield.

3. Davidsohn, I. (1969) Clinical Diagnosis by Laboratory Methods (Todd, J. C \& Sanford, A. H., eds.) 14 th Ed., S. 34, WB Saunders Co., Philadelphia.

4. Fishberg, A. M. (1954) Hyper-Tension and Nephritis, 5 th Ed., Verlag Lea and Febinger, Philadelphia.

5. Welty, J. W. (1937) Amer. J. Med. Sci. 194, 70-74.

6. King, S. E. \& Baldwin, D. S. (1956) Amer. J. Med. Sci. 20 $217-224$ konzentration wurden folgende Bereiche der quantitativen Eiweißbestimmung den Meßpunkten der Teststreifenskala zugeordnet:

$>150-300=300 \mathrm{mg} / \mathrm{l},>500-2500 \cong 1000 \mathrm{mg} / \mathrm{l}$,

$>2500-5000 \triangleq 5000 \mathrm{mg} / \mathrm{l}$.

Die Häufigkeitsverteilung der Teststreifenanzeigen ergibt in diesem Bereich 80-90\% richtige Anzeigen für den Rapignoststreifen und etwa 60-85\% richtige Anzeigen für den Comburstreifen (Abb. 2).

Insgesamt werden die Konzentrationsbereiche für semiquantitative Bestimmungen gut getrennt. Die Teststreifen zeigen allgemein richtige Resultate und eignen sich somit gut für Screeningzwecke.

7. Kutter, D. (1976) Schnelltests in der klinischen Diagnostik, S. 97, Urban und Schwarzenberg, München/Berlin/Wien.

8. Gyure, W. L. (1977) Clin. Chem. 23, 876-879.

9. Feigl, F. \& Anger, V. (1937) Mikrochim. Acta 2, 107-110.

10. Bowie, L., Smith, S. \& Gochman, N. (1977) Clin. Chem. 23, $128-130$.

11. Killingsworth, L. M., Britain, C. E. \& Woodard, L. L. (1975) Clin. Chem. 21, 1465-1468.

12. Scholer, A., unveröffentlichte Arbeit.

13. Brehler, P. (1978) Ärztl. Lab. 24, 339-345.

Prof. Dr. D. J. Vonderschmitt Klinisch-chemisches Labor Kantonsspital CH-4031 Basel, Schweiz 